\title{
MUSTIKA PAAJAH-AJAHAN PAWATEKAN SAJERONING "PUPULAN SATUA BAWAK BASA BALI" KAWIAN I GDE DHARNA
}

\author{
I Kt. Yasa ${ }^{1}$, I. B. Putra Manik Aryana ${ }^{1}$, I Kt. Paramarta ${ }^{2}$ \\ Jurusan Pendidikan Bahasa Bali \\ Universitas Pendidikan Ganesha \\ Singaraja, Indonesia \\ e-mail: \{ketutyasa150@yahoo.co.id, putramanikaryana@gmail.com, \\ ketutgembel@yahoo.co.id\}@undiksha.ac.id
}

Kuub

Tetilikan puniki matetujon nlatarang: (1) wangun jroning karya sastra, lan (2) mustika paajah-ajahan pawatekan. Jejering tetilikan, pupulan satua bawak basa Bali kawian I Gde Dharna. Penandang tetilikan, wangun jroning karya sastra, lan mustika paajah-ajahan pawatekan. Tetilikan nganggen kramaning dokumentasi, ngwacen, lan nyatet ri kala mupulang data wangun jroning karya sastra, lan mustika paajah-ajahan pawatekan. Data tureksa sane kanggen: (1) reduksi data; (2) nlatarang data; Ian (3) panyutetan (verifikasi). Pikolih tetilikan pupulan satua bawak basa Bali kawian I Gde Dharna, sakadi: (1) wangun jroning karya sastra wenten pitu, sakadi: murda, unteng, pragina, rerawatan, lelintihan, pangresep, lan piteket. (2) Mustika paajah-ajahan pawatekan kapolihang 18, sakadi: sradha bhakti, arjawa, awiwahara, tinut sesana, teleb makarya, ngawi parindikan anyar, sida ngraga, nenten minayang sesamen jana, rasa meled uning, dharma negara, tresna negara, ngajiang kawagedan, masawitra, santi rasa, anteng ngwacen, ulati pawongan, ulati palemahan, lan tinut swadharma.

Kruna jejaton: Mustika Paajah-ajahan Pawatekan, Satua Bawak.

Abstrak

Penelitian ini bertujuan menjelaskan: (1) unsur intrinsik; dan (2) nilai pendidikan karakter bangsa. Subjek penelitian, kumpulan cerpen bahasa Bali karangan I Gde Dharna. Objek penelitian, unsur intrinsik, dan nilai pendidikan karakter bangsa. Penelitian ini menggunakan metode dokumentasi teknik baca catat pada saat mengumpulkan data unsur intrinsik, dan nilai pendidikan karakter bangsa. Analisis data yang digunakan: (1) reduksi data; (2) deskripsi data; dan (3) kesimpulan/verifikasi. Hasil penelitian kumpulan cerpen bahasa Bali karangan I Gde Dharna, antara lain: (1) unsur intrinsik ada tujuh, seperti: judul, tema, tokoh, setting latar, alur, sudut pandang, dan amanat, (2) nilai pendidikan karakter bangsa ditemukan 18, seperti: religius, jujur, toleransi, disiplin, kerja keras, kreatif, mandiri, demokratis, rasa ingin tahu, semangat kebangsaan, cinta tanah air, menghargai prestasi, bersahabat, cinta damai, gemar membaca, peduli sosial, peduli lingkungan, dan tanggung jawab.

Kata kunci: Cerpen, Pendidikan Karakter Bangsa. 


\begin{abstract}
This study was aim to explain: (1) intrinsic elements; and (2) the value of character educational nation. Research subjects were collection of short stories in Bali. The objects of research were intrinsic elements, and the value of character educational nation. This research used the documentation method of reading technique. The analysis of data used: (1) data reduction; (2) data description; and (3) conclusion/verification. The result of the study of Balinese short stories collection by I Gde Dharna, among others: (1) there are seven intrinsic elements, such as: title, theme, character, setting, plot, point of view, and mandate, (2) the values of character educational nation are found 18, such as: religious, honest, tolerant, disciplined, hard work, creative, independent, democratic, curiosity, spirit of nationality, love of homeland, appreciate the achievement, friendly, peaced love, delight in reading, social caring, care of environment, and responsibility.
\end{abstract}

Keywords: Character Educational Nation, Short Stories.

\section{PURWAKA}

Sajeroning kurikulum 2013 (K13) kasaratang pisan indik paajah-ajahan pawatekan, lan kawentenane punika kabuatang antuk pemerintah. Ring media sosial/cetak akeh kapanggihin wacana indik paajah-ajahan pawatekan, punika sane ngawi sekolah makinkin lan mautsaha sajeroning paajah-ajahan nyobiahang lan nglaksanayang paajahajahan pawatekan.

Paajah-ajahan inggih punika paubahan tata papineh lan tata laksana jana saking durung midep utawi uning dados uning lan waged. Koesoema (2007:90) pawatekan kacingakin saking solah, lan parilaksana sumangdena kalimbakang majeng ring anake alit. Nasrullah (2015:483) paajah-ajahan pawatekan punika mustika kahuripan sane minayang jana sareng sarwa maurip sane lianan, jana sane madue pawatekan becik wantah sane madue rasa sutindih, subhakti, tata susila sane anut, lan budi pekerti sane luih. Prasida kacutetang paajah-ajahan pawatekan inggih punika ngeninin indik daging pikayunan, tata susila, tata papineh, tata laksana, lan bebaosan.

Pikolih tetilikan Nurgiyantoro lan Anwar warsa 2013 wenten 11 mustika paajah-ajahan pawatekan sane kapolihang, sakadi: (1) arjawa; (2) sradha bhakti; (3) tresna negara; (4) ngawi parindikan anyar; (5) anteng ngwacen; (6) tinut sesana; (7) tinut swadharma; (8) ulati palemahan; (9) teleb makarya; (10) ulati pawongan; lan (11) rasa meled uning.

Mustika paajah-ajahan pawatekan prasida kapanggihin ring satua bawak. Satua bawak ngranjing ring kasusastraan Bali anyar. Satua bawak sinalih tunggil karya sastra maosang parindikan samian kahuripan praginanyane. Tetilikan satua bawak sampun naenin kalaksanayang antuk Soetantyo warsa 2013 kasinahang kawigunan satua bawak prasida kanggen sesuluh utawi panuntun indik tata laksana para sisia ring sekolah. Sajeroning satua bawak madaging mustika paajah-ajahan pawatekan sane maguna lan mapaiketan sajeroning paajah-ajahan pawatekan ring sekolah.

Madasar saking kaluihan mustika paajah-ajahan pawatekan sajeroning satua bawak, parindikane punika sane ngawi panilik nglaksanayang tetilikan satua bawak basa Bali. Satua bawak sane katilik marupa pupulan satua bawak basa Bali kawian I Gde Dharna. Sajeroning satua bawak punika madaging mustika paajah-ajahan pawatekan sane prasida katilik. Kaluihan satua bawak I Gde Dharna rerawatan ceritanyane samian magenah ring wewidangan Buleleng. Pangawi saking Sukasada, sampun kasub indik reriptannyane, lan molihang penghargaan saking pemerintah kabupaten, provinsi marupa "Wija Kusuma", "Dharma Kusuma Madia", "Dharma Kusuma", lan polih hadiah sastra rancage. Mawiwit saking parindikane 
punika panilik rumasa garjita nilikin kawian dane.

Ngrerehin Pikobet, pikobet-pikobet sane wenten ring pupulan satua bawak basa Bali Kawian I Gde Dharna sakadi: (1) wangun jroning karya sastra; (2) wangun jaba karya sastra; (3) mustika paajahajahan pawatekan; (4) (campur kode); (5) basita paribasa miwah sane tiosan.

Ngwatesin Pikobet, tetilikan puniki nilikin kalih pikobet/variabel tetilikan. Tetilikan sane kalaksanayang wantah indik: (1) wangun jroning karya sastra lan (2) mustika paajah-ajahan pawatekan. Maka kalih pikobet punika dados pamucuk sajeroning tetilikan puniki.

Bantang pikobet tetilikan:

sapunapi wangun jroning karya sastra sajeroning pupulan satua bawak basa Bali kawian I Gde Dharna? (2) napi manten mustika paajah-ajahan pawatekan sajeroning pupulan satua bawak basa Bali kawian I Gde Dharna?

Tetujon tetilikan: (1) prasida ngwedar wangun jroning karya sastra sajeroning pupulan satua bawak basa Bali kawian I Gde Dharna; lan (2) prasida nlatarang mustika paajah-ajahan pawatekan sajeroning pupulan satua bawak basa Bali kawian I Gde Dharna.

Kawigunan pamucuk sane prasida kapolihang saking tetilikan, maguna kanggen nglimbakang widya pangweruhan basa, ngicenin pangresep paajah-ajahan indik wangun jroning karya sastra, lan mustika paajah-ajahan pawatekan. Kawigunan panglimbak sane kapolihang: (1) majeng sisia/mahasisia, tetilikan puniki ngicenin pratiwimba, widya pangweruhan indik wangun jroning karya sastra lan mustika paajah-ajahan pawatekan; (2) majeng pangwacen, ngicen pangweruhan wangun jroning karya sastra, lan mustika paajah-ajahan pawatekan; (3) majeng panilik lianan, kanggen pratiwimba olih panilik lianan sane madue arsa/pikayunan nglaksanayang tetilikan sane mapaiketan sareng tetilikan puniki; (4) majeng panilik, kanggen dasar papineh, malaksana, mabebaosan, mangda anut ring mustika paajah-ajahan pawatekan.

Arikunto (2005:78) sepat siku-siku dahat mabuat pisan duaning prasida ngicenin pamargi lan ngwantu panilik muputang tetilikannyane. Sepat siku-siku sane kanggen, sakadi: (1) tetilikan asoroh; (2) landasan teori, sakadi: kasusastraan Bali; (b) satua bawak; (c) wangun jroning karya sastra; (d) mustika paajah-ajahan pawatekan; lan (e) arti (makna); Ian (3) model tetilikan.

Kasusastraan Bali, Suwija lan Manda (2015:64) "kasusastraan" mateges kawentenan ajah-ajah sane luih/becik. Kasusastraan Bali inggih punika saluir ajah-ajahan/kaweruhan sane becik, pakaryan para wagmine ring Bali sane kasurat mabasa Bali, inggian nganggen aksara Bali lan huruf Latin.

Satua bawak ngranjing ring kasusastraan Bali anyar. Sari lan Suparsa (2011:124) satua bawak karya sastra sane bawak, nyaritayang kahuripan jana apaos manten.

Wangun jroning karya sastra, Depdiknas (2008:94) ngwedar baga wangun jroning karya sastra wenten pitu, sakadi: (1) murda; (2) unteng; (3) pragina; (4) rerawatan; (5) lelintihan; (6) pangresep; lan (7) piteket.

Mustika paajah-ajahan pawatekan, Nurgiyantoro lan Anwar (2013:384) mustika paajah-ajahan pawatekan inggih punika solah lan tata parilaksana jana sane anut ring awig (norma) sane wenten ring pakraman, sakadi spiritual, personal, sosial. Kemendiknas (2015:35-37) baga mustika paajah-ajahan pawatekan wenten 18, sakadi: (1) sradha bhakti; (2) arjawa; (3) awiwahara; (4) tinut sesana; (5) teleb makarya; (6) ngawi parindikan anyar; (7) sida ngraga; (8) nenten minayang sesamen jana; (9) rasa meled uning; (10) dharma negara; (11) tresna negara; (12) ngajiang kawagedan; (13) masawitra; (14) santi rasa; (15) anteng ngwacen; (16) ulati pawongan; (17) ulati palemahan; lan (18) tinut swadharma.

Arti (makna), arti (makna) wenten ring widya semantik. Semantik inggih punika pahan saking widya linguistik, sane malajahin indik arti (makna). Soroh arti kaepah dados, arti leksikal lan arti gramatikal. Suwandi (2008:101) paiketan arti (relasi makna) inggih punika paiketan pantaraning satuan basa sareng basa sane tiosan, sakadi: (1) sinonimi; (2) 
antonim; (3) homonimi; (4) polisemi; (5) hiponim; (6) ambiguitas; (7) redundansi. Paiketan tata arti wenten sane kawastanin hubungan kesetaraan, Sukmana lan Budiman (2010:12) hubungan kesetaraan inggih punika paiketan kalih utawi langkungan parinama (istilah) sane kaangkenin madue arti pateh (dianggap sama).

\section{KRAMANING TETILIKAN}

Wenten lelima papalih paridabdab kramaning tetilikan sakadi: (1) Pendekatan lan soroh tetilikan, tetilikan puniki ngangge pendekatan kualitatif, santukan soroh tetilikannyane ngranjing ring tetilikan kualitatif; (2) genah tetilikan, tetilikan kalaksanayang ring buku pupulan satua bawak basa Bali kawian I Gde Dharna. Buku pupulan satua bawak basa Bali kawian I Gde Dharna kapolihang ring wewidangan Singaraja (toko buku Indra Jaya); (3) soroh lan wit data; soroh data sane kapolihang marupa kruna/lengkara. Wit data kapolihang ring pupulan satua bawak basa Bali kawian I Gde Dharna. Data sane kapolihang indik wangun jroning karya sastra lan mustika paajahajahan pawatekan; (4) kramaning mupulang data, mupulang data pidabdab sane nyinahang sapunapi data kapolihang. Tetilikan kualitatif molihang data marupa kruna/lengkara. Kramaning kanggen mupulang data inggih punika kramaning dokumentasi, dokumentasi, ngwacen, lan nyatet (teknik baca catat) kanggen, santukan data pupulan satua bawak basa Bali marupa sesuratan (buku); lan (5) kramaning lan data tureksa, pidabdab sane kalaksanayang sajeroning data tureksa sakadi: (a) reduksi data; (b) nlatarang data; (c) panyutetan/verifikasi.

\section{PIKOLIH LAN TETEPASAN}

Pikolih lan tetepasan tetilikan wangun jroning karya sastra sajeroning pupulan satua bawak basa Bali kawian I Gde Dharna katlatarang ring sor puniki.

Murda satua bawak kapertama Dadong Kari. Untengnyane masawitra leket. Pragina, wenten telu likur pragina sane kapanggihin sakadi, Dadong Kari, I Wayan Rupa, Pak Kendur/Pak Wenten, I Ketut Oka/I Pacet, Dr. Ketut Nurija, I Gede Seruti, Gusti Satria, Mayor Gusti Putu Wisnu, Bapak Letkol Gusti Ngurah Rai, Luh Sadri, I Putu Astika, Serdadu Belanda, Tentara Nica, Pan Gelgel/Pan Gombrang, I Gombrang, I Made Otar, Pak Metra, Dayu Mirah, Dayu Komang, I Kadek, I Putu Sutapa, I Putu Astika, lan Patih Gajah Mada.

Rerawatan, rerawatan indik genah sakadi, di malun peken Sukasada, di Panji kelod kauh, di duur Gitgite, di rurunge, di Banjar Gintungan desa Selat, di Panji, di Banjar Tapuk Basa dangin tukad Panjine, di kubune, di Beratan, di banjar Petunge Sambangan, di Tangsi, di desa Sukasada, di kota, di daganganne Dadong Kari, kubun/umahne Dadong Kari, lan di pateluan desane. Rerawatan galah sakadi, pukul lima sabilang sanja, Tilem Sasih Karo dina Saniscara pukul kutus, ituni sanjane pukul nem, saniscara, jam dasa, setonden endag matan aine, tanggal 20 Nopember 1946, i maluan, sandikala, peteng, lan buin mani ngedas lemahang. Rerawatan kahanan sakadi, makenyem lega, sedih, jejeh, angin keras, bengong/kememegan, dingin, tangkejut, ngiki kedek, lan takut.

Lelintihan, ngangge lelintihan ka arep. Pangresep, ngangge pangresep jana kaping tiga. Piteket, dados jana mangda satata mautsaha, teleb makarya, nenten ngawi biuta, lan nenten minayang sesamen jana.

Murda satua bawak kaping kalih Tusing Ada Apa De. Untengnyane veteran durung polih gajih. Pragina, wenten limolas pragina sane kapanggihin sakadi, Bapa Wenten, I Nyoman Gatra, Ni Made Sutri, Pak Sanusi, Bapak Letkol I Gusti Ngurah Rai, I Gusti Ngurah Mayor, I Dewa Made Diksa, I Made, Ketut Mas, I Putu Subaga, Serdadu Belanda, Luh Ayu, Ni Made Gatri, I Putu Sarna, Ian Pan Sinta.

Rerawatan, rerawatan indik genah sakadi, di korsi tiingne, di paon, di kamare, di balene, di kamar mandi, di Pebantenan, ring alas, di Gunung Agung, Sekumpul, Tanah Aron, Tegal Amben Seririt, Alas Silangjanane, di korinne diwangan, di sisin 
rurunge gede, di bangkune lantang, di tegale, di dampar, di Puskesmas, di kilometer 17 Pangkung Bangka, lan di natahe. Rerawatan galah sakadi, semeng pesan sawetara pukul enem, buin lima bulanne nuju redite, sawetara pukul kutus, pukul setengah dasa, semengan, petang sasih, i maluan, malih kalih rainanne, di Sukrane, lan tengai Rerawatan kahanan sakadi, makenyem, angseg-angseg, lega, tangkejut, bagia, bengong, sedih, lan kendel pisan.

Lelintihan, ngangge lelintihan ka arep. Pangresep, ngangge pangresep jana kaping tiga. Piteket, dados jana mangda prasida ngajinin perjuangan para pejuange sane sampun nindihin gumi kantos prasida jagate merdeka.

Murda satua bawak kaping tiga Sangkur Mabet Jamprah. Untengnyane mabet sugih. Pragina, wenten solas pragina sane kapanggihin sakadi, Bapa Gede, Ni Made Kerti, I Gede Parta, I Mara, Tentara Belanda, Nica Gandek, I Dangin, I Catur, Ketut Jineng, I Mataram, lan Bapa Lingga.

Rerawatan, rerawatan indik genah sakadi, di ampikne, di natahne, di korsine, di warung, di paon, di kamar, di obagobagan jelanane, di perempatane, di rumah sakit, lan di Kantor Pemerintahan. Rerawatan galah sakadi, semengan, petang dina, i poan, limang tiban, Sukra, sanjane pukul empat, lan i maluan. Rerawatan kahanan sakadi, kebus dingin, tangkejut, mapangenan, bengong, makenyem, ngeling, sedih, sebet, lan sakit.

Lelintihan, ngangge lelintihan ka arep. Pangresep, ngangge pangresep jana kaping tiga. Piteket, dados jana mangda nenten ngulurin momo lan nenten alpaka kapining guru rupaka/rerama.

Murda satua bawak kaping pat Pocol. Untengnyane mantu bangras. Pragina, wenten telulas pragina sane kapanggihin sakadi, Men Gede, Pan Gede, I Made, I Ketut, I Nyoman, I Gede, I Ketut (kurnanne I Gede), Luh Putu, Najimudin, Nengah, Ida Bagus Ngurah, Gusti Ngurah Made, lan I Nengah Kawan.

Rerawatan, rerawatan indik genah sakadi, di balene, di kamar, di ampike, di Banjar Unasan Desa Gobleg, di pisagane, di natahe, di obag-obagan jelanan kamar, di jumahan, di kamar mandi, lan di paon. Rerawatan galah sakadi, uli itelun, peteng pukul solas, nuju perwani, pukul empat, petang bulan, selid sanja, telu bulan, sabilang peteng, pukul roras, pukul telu, i maluan, peteng, ngedas lemahang, semengan lan nyanjaang. Rerawatan kahanan sakadi, bengong, bagia, tengkejut, ngeling, gering ngangseg, sengal-sengal tur sigsigan, ujan tatit, kerug, lan galak.

Lelintihan, Ielintihan campuhan. Pangresep, ngangge pangresep jana kaping tiga. Piteket, dados jana mangda nenten ngawi biuta lan nenten bangras ri kala mabebaosan.

Murda satua bawak kaping lima Nyoman Ayu. Untengnyane lascarya nglaksanayang swadharma. Pragina, wenten pitu pragina sane kapanggihin sakadi, Ni Nyoman Ayu, Bapak Bayupati, I Ketut Jingga, Tuan Rudolf, Tentara Nica, Bung Amir, Bapa Sara.

Rerawatan, rerawatan indik genah sakadi, di markas, di umahne Nyoman Ayu, di kursine, di ampike, di tengah jalan, di batune gede, di rurunge, di ambene, di obag-obagan jelanane, di sombah kamar mandine, di duur meja makane, di samping balene, di kota, lan di tangsi Seririt. Rerawatan galah sakadi, i limang dina, sanja, sandikala, redite pukul 10, sore, sanja pukul lima, redite, Saniscara, tengai, lan ngapoan. Rerawatan kahanan sakadi, bingung, egar makenyem, bengong, bagia, kememegan tur bingbang, bagia tur kendel, kamemegan tur bengong, sedih, jejeh, lan galak.

Lelintihan, ngangge lelintihan ka arep. Pangresep, ngangge pangresep jana kaping tiga. Piteket, dados jana mangda nenten madue rasa canidra sane banget kantos mademang jana.

Murda satua bawak kaping nem Tan Pamerih. Untengnyane, kalascaryan para pejuang. Pragina, wenten pat belas pragina sane kapanggihin sakadi, I Pastima, Ida Bagus Gede, I Satia, I Kardi, Ida Ayu Sasih, I Kolar, I Brenges, I Wayan Intaran, Tentara Belanda, Nang Kepod, Bapa Korea, Bapa Widen, Bapa Jati, Nyoman Bintang. 
Rerawatan, rerawatan indik genah sakadi, di Desa Sulangga, di Munduk Saab Banjar Pebantenan, ring Banjar Selag Rurung, di desa, di Munduk Saab Puncak Landep duur Desa Panjine, di Banjar Kedu, di tampul kubune, di balebalene, di bucun munduke, di Bukit Silangjanane, di pangkunge, di selat tukad Yeh Birune, di tampule, ring Gintungan, di tengah kubune, di geria, di ambene, di pamereman, di delod desane di batan punyan poh anguse, di tanggun desane badelod, di carike, di warung, di tengah rurunge, lan di Kota Pabean. Rerawatan galah sakadi, pukul kutus semengan, pukul dasa peteng, pukul dasa liwat limolas menit, pukul dasa, imaluan, dasa rahina, lan tengah lemeng. Rerawatan kahanan sakadi, sungsut, jejeh, gedeg, jengah, makedekan,tangkejut, makenyem, bengong, kamemegan, sakit, lan kedek ngakak.

Lelintihan, ngangge lelintihan ka arep. Pangresep, ngangge pangresep jana kaping tiga. Piteket, dados jana mangda nenten malaksana iwang sakadi, dados penghianat bangsa, corah, sombong, degag, lan marikosa.

Murda satua bawak kaping pitu Pelabuhan Buleleng. Unteng, Monumen Perjuangan Yudha Mandala Tama. Pragina, wenten pitulas pragina sane kapanggihin sakadi, I Gede Ole, Nang Geloyoh, Mr. I Gusti Ketut Puja, Ida Bagus Putra Manuaba, Kapten I Gede Muka Pandan, Kapten I Dewa Suwija, Letnan Wayan Mudana, Letnan Anang Ramli, Pak Ketut Wijana/Pak Item, I Gusti Ngurah Rai, Mayor Anami, Ketut Merta, Chookang, Serdadu Jepang, Putu Gatra, Ni Nyoman Tantri, lan Ni Made Sayang.

Rerawatan, rerawatan indik genah sakadi, Pelabuhan Buleleng, ring sor kayu Ketapange, di Pempatan dajan peken Anyare, di malun umah Chookange, di kantor bupatine, di gelebege, di malunmalun tokone, di umah-umahe sisin rurunge, dangin soan durin gudange, di durin Pura Segarane, di rurunge, lan ring tepin segarane kauh. Rerawatan galahsakadi, nuju Saniscara, pukul setengah lima sore, pukul dasa, tanggal 8 Oktober 1945, tanggal 17-8-1945, tanggal 25 Oktober 1945, pukul 10 semengan, sabilang wai, tanggal 26 Oktober 1945, lan tanggal 27 Oktober 1945. Rerawatan kahanan sakadi, tengkejut, suryak-suryak, lan brangti.

Lelintihan, Ielintihan campuhan. Pangresep, pangresep jana kaping tiga. Piteket, dados jana mangda sida tresna asih lan madue rasa seneng sajeroning nelebin sejarah.

Pikolih lan tetepasan tetilikan mustika paajah-ajahan pawatekan pupulan satua bawak basa Bali kawian I Gde Dharna 18 sane kapolihang, katlatarang ring sor puniki.

Sradha bhakti, kapanggihin ring satua bawak sane mamurda Pocol. Data sane kapolihang sakadi puniki.

"Inget teken dewekne kalahina Pan Gede bergerilya sedeng melingang pianakne I Gede mara petang bulan. Selid sanja Men Gede ngaturang bunga di Kemulan, nunas ring ida sumangden Pan Gede selamet".

Sajeroning hiponim, sradha bhakti kacihnayang antuk parilaksana sakadi: ngaturang bunga, mabanten saiban, ngejot wedang. Men Gede sampun nglaksanayang ajaran agama. Men Gede selid sanja ngaturang bunga di Kemulan sumangdena Pan Gede selamet.

Sradha bhakti kacihnayang antuk parilaksana ngaturang bunga ring sanggah kemulan, lan nunas ica ring Ida Sang Hyang Widhi Wasa. Men Gede nglaksanayang mustika paajah-ajahan pawatekan sradha bhakti malarapan antuk selid sanja ngastiti bhakti mangda manggih karahayuan.

Arjawa, kapanggihin ring satua bawak sane mamurda Nyoman Ayu. Data sane kapolihang sakadi puniki.

Sambilanga nutur tusing asena suba nganti nemnem tuan rudolf nelahang jaja. Sambilanga kedek ia mamunyi, "Karena keenakan, tidak terasa sudah enam biji pisang gorengnya saya habiskan".

"Tidak apa-apa, memang saya buat ini hanya untuk tuan", $\mathrm{Ni}$ Nyoman Ayu masaur sinambi makenyem.

Manut arti gramatikal, parilaksana tuan Rudolf arjawa, kasinahang antuk 
dane ngangkenin yening sampun nenem ngajeng pisang gorengne Nyoman Ayu. Bebaos tuan Rodulf sane nyambat sara sampun akeh ngajeng pisangne Nyoman Ayu pinaka cihna yening dane arjawa, yadiastun sampun akeh ngajeng pisang, nanging nenten kemad jani ngorahang ring Nyoman Ayu.

Parilaksana pongah juari saking tuan Rodulf wantah utsaha dane mangda prasida sida angganyane satata kagugu sajeroning wacana kapining $\mathrm{Ni}$ Nyoman Ayu. Ni Nyoman Ayu ngajinin bebaos, Ian laksana tuan Rodulf sane arjawa ngangkenin sampun akeh ngajeng pisang gorengne. Punika cihna yening tuan Rudolf madue mustika paajah-ajahan pawatekan arjawa ri kala magenduwirasa sareng Nyoman Ayu.

Awiwahara, kapanggihin ring satua bawak sane mamurda Pelabuhan Buleleng. Data sane kapolihang sakadi puniki.

Dinane ento Komandan BKR, ngaukin Kapten Muka Pandan, Letnan Anang Ramli, Letnan Wayan Mudana. Dugase ento kabenengan pesan Pak Ketut Wijana alias Pak Item, mara gati teka uli majalan batis uli Tabanan ngajak pasukan, lakar bareng sutindih teken Bali. Keto masi dane I Gusti Ngurah Rai durinan rauh uli Badung, masi ngajak rombongan. Petemuane dugase ento mutusang sumangden petenge ngadaang gerakan nuunang bendera Belandane, nganti aji merah putih.

Manut arti gramatikal, para pejuange yadiastun mabinayan agama, kasta, nanging prasida masikian sajeroning parum/maremug makinkin ngwentenang pangrencana nedunang bendera Belandane.

Para pejuange wenten sane saking dura Bali, wenten sane pragusti, jaba lan jabatanne matios-tiosan, nanging para pejuange prasida masikian satya nindihin jagat Bali. Parilaksana para pejuange sane prasida narima pabinayan agama, kasta, lan ngajinin parilaksana pejuang sane lianan, kasengguh para pejuange madue mustika paajah-ajahan pawatekan awiwahara sajeroning kahuripannyane.
Tinut sesana, kapanggihin ring satua bawak sane mamurda Sangkur Mabet Jamprah. Data sane kapolihang sakadi puniki.

Madingehang pamunyin panakne buka keto, Bapa Gede kedek di kenehne. Masaut lantas ia banban tur alus, "Ooo, keneh caine apang bapa ngadep jabatan bapane, keto? Enden malu, Gede. Adenan bapa mati makenta ngajak cai lan adine, padaan cai nunden bapa ngalih daar majalaran ngadep jabatan. Depang anake lenan malaksana buka keto. Bapa takut teken sumpah jabatan bapane dugas lantika dadi pegawe, takut teken karma pala, sawireh bapa ngayasaang cai ajak adine. Mani poan apang tusing cai ningeh pamunyin anak dirurunge, ngelahe ban bapane korupsi. Di aape dong saja tusing ada anak juari ngomong. Sakewala tundune ajak liu nulisin".

Manut arti gramatikal, Bapa Gede anut ring tata parilaksana sajeroning dados pejabat, kacihnayang antuk parilaksana Bapa Gede sane becik eling ring sumpah jabatan.

Bapa Gede takut yening prade korupsi kapungkuran dados orta ring pakraman. Bapa Gede sajeroning dados pejabat sampun tinut sesana sajeroning makarya nenten kayun ngadep jabatan sane sampun kapolihang. Malarapan kawentenane punika, Bapa Gede madue mustika paajah-ajahan pawatekan tinut sesana.

Teleb makarya, kapanggihin ring satua bawak sane mamurda Sangkur Mabet Jamprah. Data sane kapolihang sakadi puniki.

Sujatinne Bapa Gede dadi pegawe negeri suba gede pangkatne, sawireh dugas revolusine ia dadi manggala, buina ia mula saja dueg. Lenan teken ento Bapa Gede polos pesan dadi pegawe tusing nyak ngadep jabatanne anggona ngalih pipis. Ento masih ane ngeranayang idupne asaksanayan pesan.

Manut arti gramatikal, yadiastun Bapa Gede daweg revolusine dados manggala, sakewanten Bapa Gede satata kukuh/anteng makarya nenten nyak 
ngadep jabatanne anggena ngrereh jinah. Punika maka cihna yening Bapa Gede madue mustika paajah-ajahan pawatekan teleb makarya sajeroning kahuripannyane, yadiastun jabatanne sampun tegeh nanging nenten nyak ngulah aluh ngadep jabatane. Bapa Gede satata ngutamayang kabecikan sajeroning dados jana anteng makarya.

Ngawi parindikan anyar, kapanggihin ring satua bawak sane mamurda Dadong Kari. Data sane kapolihang sakadi puniki.

I maluan dugas Dadong Kari enu ceteng, gaginane madagang matanja ka desa-desa, ngadep isin paon buka sera, uyah, asem, be gerang, pindang, muah bajo. Kacang manyahnyahne Dadong Kari kasub pesan teken rempuh turmaning renyah. Tusing nyamanyamane di Sukasada dogen ane ngalaisang, sakewala jantos ka kota.

Manut arti gramatikal, kasinahang Dadong Kari daweg dumun kantun ceteng geginane matanja ka desa-desa, ngadep isin paon buka sera, uyah, asem, be gerang, pindang, muah bajo, duaning sampun lingsir mautsaha ngawi usaha anyar inggih punika dados dagang kacang, yadiastun dados dagang kacang nenten ja pakaryan sane anyar, sakewanten Dadong Kari ngadol kacang manyahnyah sane rempuh pisan, ngadol kacang manyahnyah sane rempuh kasengguh ngawi parindikan anyar duaning daweg punika durung wenten sane ngadol kacang manyahnyah rempuh. Manut kawentenane punika, Dadong Kari madue mustika paajah-ajahan pawatekan ngawi parindikan anyar.

Sida ngraga, kapanggihin ring satua bawak sane mamurda Pocol Data sane kapolihang sakadi puniki.

"Pan Gede anak mula tusing ngelah nyen. la lekad buka batun buluane, ngelecir padidiana pesan. Meme bapanne suba makelo ngalahin, senun Pan Gedene Bajang. Buina ia lekad di tanahe perangan, tuh kaang".

Manut arti gramatikal, Pan Gede saking alit sampun nenten madue rerama nglintik buka batun buluane, nika sane ngawi Bapa Gede sida ngraga sajeroning kahuripannyane.

Parilaksana sane nenten muatang wantuan jana siosan, sakadi parilaksana Bapa Gede kasengguh jana madue mustika paajah-ajahan pawatekan sida ngraga, santukan Bapa Gede nenten satata nyagerang jana siosan ri kala muputang pakaryan ring kahuripannyane.

Nenten minayang sesamen jana, kapanggihin ring satua bawak sane mamurda Nyoman Ayu. Data sane kapolihang sakadi puniki.

"Sakewala Ni Nyoman nyanggra tur ngayahin para pejuange ane singgah di umahne tusing taen mabinayan".

Sajeroning hubungan kesetaraan, lengkara "nyanggra tur ngayahin para pejuange ane singgah di umahne tusing taen mabinayan" kaangkenin madue arti pateh sareng "nenten minayang sesamen jana".

Ni Nyoman Ayu nenten minayang padewekannyane sareng jana sane tiosan. Ni Nyoman nyanggra tur ngayahin para pejuange sane singgah ring umahne nenten mabinayan, nika maka cihna yening Ni Nyoman Ayu madue mustika paajah-ajahan pawatekan nenten minayang sesamen jana sajeroning kahuripannyane.

Rasa meled uning, kapanggihin ring satua bawak sane mamurda Pelabuhan Buleleng. Data sane kapolihang sakadi puniki.

"Kaki, kayu-kayune ane majujuk ento lad apa sih?, I Gede Ole mataken sinambi matujuang." I Gede Ole matakon malih, "Kija kirima sampi, celeng, kopi pada?"

Manut arti gramatikal, parilaksana I Gede Ole sane matakon kapining kakinne maka cihna madue rasa meled uning ring parindikan sane kacingakin. Rasa meled uning kacihnayang antuk parilaksana sane satata mautsaha mangda prasida uning parindikan sakadi sane kacingakin, maka duaning I Gede nyingakin kayu-kayu sane majujuk ring Pelabuhan, ri kala punika I Gede mamanah mangda tatas uning indik kasujatian kayune punika.

Siosan ring punika, I Gede malih mataken ring kakinne indik sampi, celeng, 
kopi baktane ring dija manten. Kakinne nyaritayang napi sane katakenin sareng I Gede. Kawentenan I Gede sane seneng mataken, maka cihna I Gede madue mustika paajah-ajahan pawatekan rasa meled uning.

Dharma negara, kapanggihin ring satua bawak sane mamurda Tusing Ada Apa De. Data sane kapolihang sakadi puniki.

Ento ada timpal veteran ane madan Pak Sanusi, uli Patas, Kecamatan Gerokgak. Apang bapa nawang, ia bareng-bareng ajaka Bapak Letkol I Gusti Ngurah Rai ka Jawa ngalih senjata, ia bareng long march ka Gunung Agung, bertempur ditu di Sekumpul, di Tanah Aron, nyegat kapten serdadu Belandane di Tegal Amben Seririt, kayang jani masi tonden teka eska maan gajihne.

Manut arti gramatikal, Pak Sanusi sareng Bapak Letkol I Gusti Ngurah Rai madue parilaksana sane sutindih lan ngutamayang panegara kacihnayang antuk dane ka Jawa ngrereh senjata, sareng long march ka Gunung Agung, bertempur di Sekumpul, di Tanah Aron, nyegat kapten serdadu Belandane di Tegal Amben Seririt.

Yadiastun Pak Sanusi saking Patas Kecamatan Gerokgak, malarapan rasa semangat sane ageng lan ngutamayang panegara mangda prasida merdeka, sareng raris dane matempur long march ka Gunung Agung. Madasar antuk rasa dharma negara sane ngawi Pak Sanusi sareng Bapak Letkol I Gusti Ngurah Rai matempur ring Tanah Aron lan nyegat kapten serdadu Belandane di Tegal Amben Seririt. Manut kawentenane punika, Pak Sanusi sareng Bapak Letkol I Gusti Ngurah Rai madue mustika paajahajahan pawatekan dharma negara, kacihnayang antuk sang makakalih dados pejuang nindihin jagat lan ngutamayang panegara mangda sida merdeka.

Ngajiang kawagedan, kapanggihin sajeroning satua bawak sane mamurda Pelabuhan Buleleng. Data sane kapolihang sakadi puniki.

"Tengkejut pesan ane nglaksanayang tugas nureksain, ninggalin timpalne makakeb maurab getih. Mara badinganga, sajaan suba Ketut Merta uli Liligundi ane mati. la ane dadi tumbal kapertama mati sangkan kawangun Monumene di Pelabuhan ento". I Kaki matujuang monumene badangin, ane suba liu ada anak ditu, lakar mabalih matan aine leb.

Manut arti gramatikal, pejuange sane padem ring Pelabuhan inggih punika mawasta Ketut Merta saking Liligundi dados tumbal kapertama. Mawit saking kawentenane punika, parajanane madue rasa ngajiang kawagedan pejuange sane padem malarapan antuk ngwangun Monumen perjuangan ring Pelabuhan.

Parilaksana parajana sane narima lan ngangkenin utsaha perjuangan para pejuange sane sampun mautsaha nindihin gumi kantos ngemasin padem, kasengguh parajana punika ngajiang kawagedan lan pikolih para pejuange. Parajanane nglaksanayang mustika paajah-ajahan pawatekan ngajiang kawagedan, macihna kawangun raris Monumen Perjuangan Yudha Mandala Tama ring Pelabuhan Buleleng.

Masawitra, kapanggihin ring satua bawak sane mamurda Nyoman Ayu. Data sane kapolihang sakadi puniki.

"Terimakasih Bung Amir". Ni Nyoman Ayu ngencolang tindakanne majalan. Bung Amir majujuk bengong ningalin pakalahne Ni Nyoman Ayu jantos ilang adegne di rurunge mamengkol. Bagia pesan kenehne prasida mapaomongan padaduanan ngajak Ni Nyoman Ayu turmaning maisi pangidihne.

Manut arti gramatikal, Bung Amir lan Nyoman Ayu masawitra, kacihnayang antuk sang makakalih magenduwirasa, tur pangidihne Bung Amir prasida kadagingin antuk Nyoman Ayu.

Bung Amir bagia pisan sampun prasida magenduwirasa sareng Nyoman Ayu, sapunika taler Nyoman Ayu ngaturang rasa suksma ring Bung Amir, indike punika maka cihna yening sang makakalih sampun masawitra leket pisan prasida paras-paros, pantaraning Bung Amir lan Nyoman Ayu sampun madue mustika paajah-ajahan pawatekan masawitra. 
Santi rasa, kapanggihin ring satua bawak sane mamurda Dadong Kari. Data sane kapolihang sakadi puniki.

"Yadiastun keto tusing ada ane sebet, sawireh Dadong Kari bisa ngaba dewek, tusing taen sinted teken anak mabelanja, yadiastun anake mabelanja muaka ngojog I Dadong tur morahan nganggeh. Buina I Dadong tusing taen gedeg yening candaina teken bajangbajange".

Manut arti gramatikal, kasinahang kawentenan Dadong Kari bisa ngaba dewek, tusing taen sinted teken anak nganggeh, taler nenten naenin gedeg yening candaina sareng bajang-bajange, maka cihna parilaksana Dadong Kari santi rasa sajeroning kahuripannyane.

Lengkara "Tusing taen sinted", "Tusing taen gedeg" bersinonim sareng "santi rasa". Santukan parilaksana tusing taen sinted, lan tusing taen gedeg maka cihna parilaksana sane ngawi karahayuan.

Anteng ngwacen, kapanggihin ring satua bawak sane mamurda Dadong Kari. Data sane kapolihang sakadi puniki.

I Wayan buin mamunyi, "Oooo

saja. Di buku "Bali Berjuang"-e maan kabaca unduk Perang Puputane di Marga ento. Tanggal 20 Nopember 1946. Dugase ento Bapak Letkol. I Gusti Ngurah Rai, Mayor I Gusti Putu Wisnu gugur sareng Pasukan Ciung Wenarane. Liunne ane gugur dugase ento ajaka 96. Yan manut di bukune siat Puputan Margaranane ento rame pesan. Serdadu Belandane mulane kalilih. Lantas ia nekaang wantuan kapal terbang uli Surabaya. Kapal terbange ene suba ngiber sambilanga nembakin, ngulungang bom, gas air mata uli baduur. Yan tusing ada kapal terbange ento, pasti kalah Belandane."

Manut arti gramatikal, I Wayan polih ngwacen buku "Bali Berjuang" indik Perang Puputane di Marga Tanggal 20 Nopember 1946. Kawentenane punika maka cihna I Wayan madue rasa seneng ngwacen malajahin parindikan mangda molihang pikolih.
Pikolih I Wayan anteng ngwacen prasida nguningin indik Perang Puputan Margaranane, daweg I Gusti Ngurah Rai, Mayor I Gusti Putu Wisnu gugur sareng Pasukan Ciung Wenarane. Maka cutetnyane I Wayan sampun anteng ngwacen mangda molihang pikolih marupa widya pangweruhan indik Perang Puputan Margarana. Manut kawentenane punika, I Wayan madue mustika paajahajahan pawatekan anteng ngwacen.

Ulati pawongan, kapanggihin ring satua bawak sane mamurda Dadong Kari. Data sane kapolihang sakadi puniki.

"Dadong inget pesan tekening Pan Kendur, dane sayang tur dana pesan teken Dadong. Yen dane rauh uling Jerone di Penataran, musti singgah di kubun Dadonge tur ngicen Dadong pipis".

Sajeroning hiponim, ulati pawongan kacihnayang antuk, (wantuan dana/jinah, wantuan barang/alat, wantuan pakaian, wantuan ubad, wantuan sembako). Manut kawentenane punika, Pak Kendur madue mustika paajah-ajahan pawatekan ulati pawongan kacihnayang antuk yening dane rauh uling Jerone di Penataran, musti singgah di kubun I Dadong tur ngicen jinah. Wantuan marupa jinah punika sane nyinahang Pak Kendur ulati pawongan kapining Dadong Kari. Wantuan jinah sane kaicen olih Pak Kendur majeng Dadong Kari maka cihna yening Pak Kendur ulati pawongan sajeroning kahuripannyane.

Ulati palemahan, kapanggihin ring satua bawak sane mamurda Tusing Ada Apa De. Data sane kapolihang sakadi puniki.

"Di natahne katanem punyan buluan telung puun, keto masi taneman bunga-bungaan. Hias pesan baane ngatur natahne. Tongos umahne Bapa Wenten di tegehe. Bapa Wenten di tegale mulungin punyan buluan, wenten nem dasa puun".

Manut arti gramatikal, Bapa Wenten ulati palemahan maka cihna di natahne katanem punyan buluan, bunga-bungaan. Siosan ring punika, natahne taler asri pisan. Bapa Wenten di tegalne mulungin punyan buluan, wenten sawetara nem dasa puun. Punika maka cihna Bapa 
Wenten madue mustika paajah-ajahan pawatekan ulati palemahan, santukan sampun prasida miara palemahannyane sakadi karesikan ring natahnyane, ngangge palemahan mangda maguna ring kahuripan, lan kacihnayang antuk nanem bunga/woh-wohan ring tegalne.

Tinut swadharma lan Tresna negara, kapanggihin ring satua bawak sane mamurda Tan Pamerih. Data sane kapolihang sakadi puniki.

Ida Ayu Sasih sedek nenun, sambilang ida magending. Mara telung baris ida mapupuh ginada, nangis okane ane ketutan, mara mayusa lima bulan. Gagesonan Ida Ayu matangi ngaranjing mulihan. Di pamereman kalanturang pupuh ginada jaya pranane jantos okane sirep buin. Di subane okane leplep sirepne, mawali Ida Ayu Sasih nutugang nenun.

Manut arti gramatikal, Ida Ayu Sasih sayang lan anteng pisan ngempu okanne sane kantun alit. Swadharmaning dados biang sinah madue rasa tinut swadharma ngupapira okanyane. Parindikane punika maka cihna yening Ida Ayu Sasih madue mustika paajah-ajahan pawatekan tinut swadharma ngupapira mangda okanyane dados suputra. Ida Ayu Sasih taler makarya tenunan, ri kala nenun yening okanyane nangis tetunane kararianang, kalanturang malih yening okanne sampun sirep. Indike punika maka cihna yening Ida Ayu Sasih satata eling dados biang patut ngupapira okanyane.

Manut arti gramatikal, Parilaksana Ida Ayu Sasih ri kala nenun sambilanga matembang pupuh ginada maka cihna dane madue mustika paajah-ajahan tresna negara, dane prasida ngajinin budaya Bali sakadi matembang pupuh ginada. Indike punika maka cihna yening Ida Ayu seneng ngukuhang budaya Bali malarapan antuk matembang ginada jayaprana. Jana sane ngajinin budaya kabaos jana punika ngajinin tetamian sane wenten ring panegaranyane. Siosan ring punika, Ida Ayu sasih taler ngajinin rabinne ri kala dados pejuang nindihin panegara mangda sida merdeka.

Tetilikan wangun jroning karya sastra lan mustika paajah-ajahan pawatekan sajeroning karya sastra sujatinnyane sampun kalaksanayang olih panilik lianan. Mawit saking kawentenane punika panilik nyaihang pikolih lan tetepasan tetilikan puniki sareng tetilikan panilik lianan, sakadi: (1) tetilikan $\mathrm{Ni}$ Kadek Juniari, kapolihang pitu wangun jroning karya sastra, dadosnyane pateh sareng tetilikan puniki. Satua Ni Daha Mayus kapolihang papat pikenoh paajahajahan pawatekan, lan satua $\mathrm{Ni}$ Wayan Sedih kapolihang akutus pikenoh paajahajahan pawatekan; (2) tetilikan Ni Putu Nova Kartika Sari, kapolihang pitu wangun jroning karya sastra, dadosnyane pateh sareng tetilikan puniki. Mustika paajahajahan pawatekan kapolihang 14, yening tetilikan puniki kapolihang 18.

Manut kawentenan ring ajeng, sesampune kasaihang pantaraning tetilikan puniki sareng tetilikan Ni Kadek Juniari lan Ni Putu Nova Kartika Sari prasida kawedarang pabinayannyane sakadi: (1) tetilikan puniki akehan molihang mustika paajah-ajahan pawatekan ring karya sastra satua bawak; (2) ri sampune panilik nguratiang tetepasannyane, ri kala nlatarang data $\mathrm{Ni}$ Kadek Juniari lan Ni Putu Nova Kartika Sari nenten nganggen widya tata arti; (3) tetilikan Ni Kadek Juniari Ian Ni Putu Nova Kartika Sari nenten pupulan satua bawak, tetilikan puniki nilikin pupulan satua bawak; (4) tetilikan puniki ngangge widya arti nlatarang mustika paajah-ajahan pawatekannyane.

Tetilikan ngangge widya arti sajeroning nlatarang mustika paajahajahan pawatekan kasengguh tetilikan sane anyar krana durung wenten sane nglaksanayang. Sayuaktinnyane, widya arti mabuat kanggen turmaning madue kaluihan ngawi tetilikan sayan jangkep lan tata cara nlatarang datanyane matiosan sareng tetilikan panilik lianan.

\section{PAMUPUT}

Prasida kacutetang wangun jroning karya sastra sajeroning pupulan satua bawak basa Bali kawian I Gde Dharna wenten pitu sakadi: (1) murda; (2) unteng; 
(3) pragina; (4) rerawatan; (5) lelintihan;

(6) pangresep; lan (7) piteket.

Mustika paajah-ajahan pawatekan sajeroning pupulan satua bawak basa Bali kawian I Gde Dharna wenten 18 sakadi: (1) sradha bhakti; (2) arjawa; (3) awiwahara; (4) tinut sesana; (5) teleb makarya; (6) ngawi parindikan anyar; (7) sida ngraga; (8) nenten minayang sesamen jana; (9) rasa meled uning; (10) dharma negara; (11) tresna negara; (12) ngajiang kawagedan; (13) masawitra; (14) santi rasa; (15) anteng ngwacen; (16) ulati pawongan; (17) ulati palemahan; lan (18) tinut swadharma.

Piteket kawedarang majeng ring: (1) sisia/mahasisia mangda nguratiang satua bawak basa Bali, madaging kaluihan mustika paajah-ajahan pawatekan sane patut kanggen sesuluh lan kalaksanayang; (2) sang sane ngwacen mangda sayan urati, lan mautsaha nglestariang tetamian panglingsire indik satua bawak. Satua bawak madaging kaluihan, pangresep wangun jroning karya sastra, lan mustika paajah-ajahan pawatekan; (3) panilik lianan mangda sida nglimbakang malih daging tetilikan sane mapaiketan sareng basa Bali, utaminnyane wangun jroning karya sastra lan mustika paajah-ajahan pawatekan.

\section{KAPUSTAKAAN}

Anindyarini, Atikah lan Sri Ningsih. 2008. Bahasa Indonesia. Jakarta: Pusat Perbukuan Departemen Pendidikan Nasional.

Arikunto, Suharismi. 2005. Manajemen Penelitian. Jakarta: PT Rineka Cipta.

Juniari, Ni Kadek. 2015. Seseleh Wangun lan Pikenoh Pendidikan Karakter ring Satua Ni Daha Mayus lan Ni Wayan Sedih. Skripsi (nenten kamijilang). Jurusan Pendidikan Bahasa Bali, FBS Universitas Pendidikan Ganesha.

Koesoema, A Doni. 2007. Pendidikan Karakter Strategi Mendidik Anak di Zaman Global. Jakarta: Grasindo.
Nasrullah, Feri Jon. 2015. "Pendidikan Karakter pada Anak dan Remaja". Malang: Jurusan Magister Psikologi, Universitas Muhamadiyah Malang. Tersedia pada http://mpsi.um.ac.id/files/file/483486 \%20Feri\%20Jon.pdf. (diakses tanggal 28 Desember 2016).

Nurgiyantoro, Burhan lan Anwar efendi. 2013. "Prioritas Penentuan Nilai Pendidikan Karakter Dalam Pembelajaran Sastra Remaja". Cakrawala Pendidikan Jurnal, Volume XXXII, Nomor 3 (kaca.382393).

Sari, Ni Made Purnami lan I Nyoman Suparsa. 2011."Peningkatan Kemampuan Menganalisis Unsur Intrinsik Cerpen Melalui Metode Diskusi Jenis Buzz Group Pada Siswa Kelas VIII A SMP Dwi Jendra Gianyar Tahun Pelajaran 2011/2012". Jurnal Santiaji Pendidikan, Volume 3, Nomor 2 (kaca.123-131).

Sari, Ni Putu Nova Kartika. 2015. Seseleh Wangun lan Pikenoh Pendidikan Karakter Cerpen Katemu ring Tampak Siring ring Modul Pendidikan Basa Daerah Bali SMA Kelas $X$. Skripsi (nenten kamijilang). Jurusan Pendidikan Bahasa Bali, FBS Universitas Pendidikan Ganesha.

Soetantyo, Sylvia Primulawati. 2013. "Peranan Dongeng Dalam pembentukan Karakter siswa Sekolah Dasar". Jurnal Pendidikan, Volume 14, Nomor 1 (kaca.44-51).

Sukmana, Ena lan Lukman Budiman. 2010. "Hubungan Semantik". Tersedia pada klikeazy.blogspot.co.id/2011/01/hub ungan-semantik.html?m=1. (diakses tanggal 27 Desember 2016).

Suwandi, Sarwiji. 2008. Semantik Pengantar Kajian Makna. Yogyakarta: Media Perkasa.

Suwija, I Nyoman lan I Gede Manda. 2015. Widia Sari Basa lan Sastra Bali 3. Denpasar. 